\title{
Framework for Face Detection
}

\author{
Nidhi Srivastava \\ Amity Institute of Information \\ Technology \\ Amity University \\ Lucknow, India
}

\author{
Harsh Dev, PhD \\ PSIT College of \\ Engineering \\ Lucknow, India
}

\author{
S. Qamar Abbas, PhD \\ Ambalika Institute of \\ Management \& Technology \\ Lucknow, India
}

\begin{abstract}
This paper shows an approach to the detection and identification of human faces in a color image. Detection of human faces is becoming a very important task in various applications, such as video surveillance and security control system, intelligent human computer interface, face recognition, content-based image retrieval, multimedia applications on the web like video conferencing and face database management. In this paper, k-means clustering has been used for distinguishing skin from non skin region. Then, some morphological operations have been performed to clean the image. Finally, region property measures have been used to locate face in the image.
\end{abstract}

\section{General Terms}

Skin Segmentation; Color; Detection; Connected;

\section{Keywords}

Face Detection; K-means; Cluster analysis; Skin color segmentation; Framework;

\section{INTRODUCTION}

Face detection is a process that aims to locate a human face in an image. The task of face detection is so trivial for the human brain, yet it still remains a challenging and difficult problem to enable a computer to do face detection [1]. This is because human face is a dynamic object and has a high degree of variability in its appearance, which makes face detection a difficult problem. The challenges associated with face detection can be attributed to factors like posture of face, facial expression and face occlusion, presence or absence of facial features such as beards, mustaches and glasses which increases the variability of facial appearance together with shape, color and size [2].

Face detection is a crucial technology for applications such as face recognition, automatic lip-reading, and facial expression recognition (Pentland, Moghaddam, \& Starner, 1994; Donato, Bartlett, Hager, Ekman, \& Sejnowski, 1999) [3].

\section{PROPOSED FRAMEWORK FOR FACE DETECTION}

In recent years, images containing human faces are becoming more and more important in different security and intelligent systems. This is the main reason why human face related topics, say, face detection, face recognition, expression recognition, emotion recognition, pose recognition, and so on, have attracted much attention of many researchers [4].

In this paper we have proposed a novel framework for face detection. In this framework, the input image is segmented to classify the skin and non skin color area. Then, all areas which classify as skin are given a value of 1 and rest of the areas is given a value of 0 . So, we get the binary image. Then morphological operations are applied on this binary image so as to get a cleaner image. Further, region based property measures are applied to detect face from this image.

Diagrammatically, the framework referred to for detection of faces in this paper is shown in Fig. 1

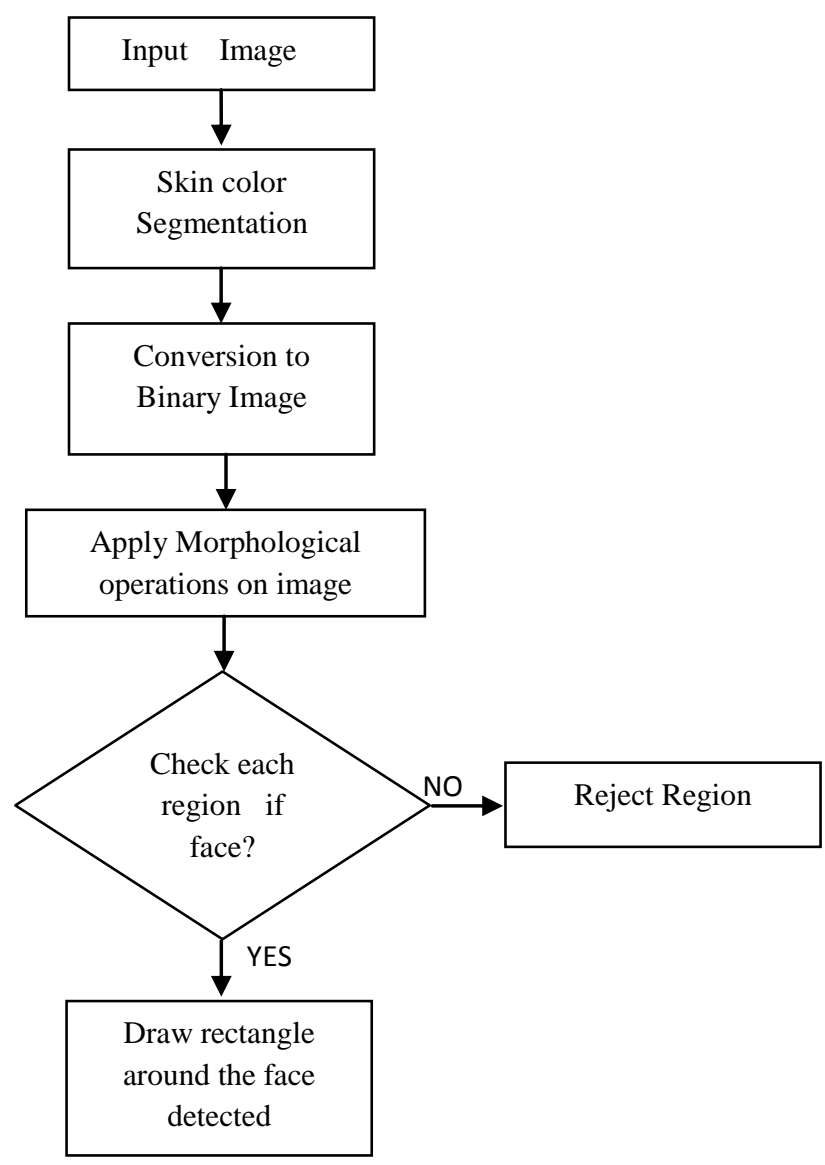

Fig 1: Face Detection Framework

\subsection{Skin Color Segmentation}

Skin detection is the process of finding skin-colored pixels and regions in an image or a video. This process is typically used as a preprocessing step to find regions that potentially have human faces and limbs in images.

Detecting skin regions is a first step in the majority of recent face detection methods. The key quality that differentiates skin from non-skin regions in images is color. Color is a powerful cue that can be used as a first step in the process of 
skin detection in images. Skin color identification (detection) can be used as complimentary information to other features to build accurate face or hand detection in recent works [5]. Various approaches to face detection are discussed in $[6,7]$.

Color is a useful piece of information for skin detection. The skin detection is the most common and first approach for detecting meaningful skin color, skin color detection may avoid exhaustive search for faces in an entire image. In this step, we describe that how non skin color is rejected from an image so that the image may contain only skin like areas, which will be our skin color segmented image for further processing [8]. Various colors spaces provides various discriminality between skin pixels and non-skin pixels over various illumination conditions. Skin color models that operate only on chrominance subspaces such as the $\mathrm{Cb}-\mathrm{Cr}$ and $\mathrm{H}-\mathrm{S}$ have been found to be effective in characterizing various human skin colors. Peer et al. constructed a set of rules to describe skin cluster in RGB space while Garcia and Tziritas used a set of bounding rules to classify skin regions on both YCbCr and HSV spaces [9]. Various color spaces have been used by different people to classify or segment the skin region. Few of the color spaces used by different authors earlier are given in Table 1.

\section{Table 1. Different Color Spaces used by authors earlier}

\begin{tabular}{|l|l|l|}
\hline Author & Year & Color Space \\
\hline $\begin{array}{l}\text { Hongming Zhang, } \\
\text { Debin Zhao, Wen } \\
\text { Gao, Xilin Chen } \\
{[12]}\end{array}$ & 2000 & YUV \\
\hline $\begin{array}{l}\text { Sanjay Kr. Singh, D. } \\
\text { S. Chauhan, Mayank } \\
\text { Vatsa, Richa Singh } \\
\text { [13] }\end{array}$ & 2003 & \\
\hline $\begin{array}{l}\text { C. N. Ravi Kumar, } \\
\text { Bindu .A [14] }\end{array}$ & 2006 & RGB, YCbCr, HSI \\
\hline $\begin{array}{l}\text { Krishnan } \\
\text { Nallaperumal, Ravi } \\
\text { Subban, } \\
\text { Krishnaveni. K, } \\
\text { Lenin Fred, } \\
\text { R.K.Selvakumar [1] }\end{array}$ & 2006 & \\
\hline $\begin{array}{l}\text { Devendra Singh } \\
\text { Raghuvanshi, } \\
\text { Dheeraj Agrawal [8] }\end{array}$ & 2012 & YCbCr \\
\hline $\begin{array}{l}\text { Zulkifli Musa, Marriran Jumari } \\
\text { Kasmirasharuddin } \\
\text { and } \\
\text { Zainal [15] }\end{array}$ & & YCbCr \\
\hline
\end{tabular}

To separate skin color from non-skin color we have made use of K-means cluster analysis.

Cluster analysis is one of the basic tools for exploring the underlying structure of a given data set and is being applied in a wide variety of engineering and scientific disciplines such as medicine, psychology, biology, sociology, pattern recognition, and image processing. The primary objective of cluster analysis is to partition a given data set of multidimensional vectors (patterns) into so-called homogeneous clusters such that patterns within a cluster are more similar to each other than patterns belonging to different clusters [10]. Clusters may be described as connected regions of a p-dimensional space containing a relatively high density of points, separated from other such regions by a region containing a relatively low density of points. The k-means clustering is an algorithm to classify object based on features or attributes into k number of group. The group is done by minimizing the sum of squares of distance likely Euclidean Distance between data and the corresponding cluster centroid [11]. K-means is widely used because of its simplicity and speed.

The number of classes affects the classification result. If $\mathrm{K}$ is too small, the desired "skin color" might be classified into a class with other "non-skin color"; if $\mathrm{K}$ is too large, "skin color" might be classified as more than one single class which results that no one class can represent the skin color exclusively. To choose a proper $\mathrm{K}$ is crucial to get a classification which is good enough for the following identification work. Experiments on other images that contain human skin content also show that the results are satisfactory when $\mathrm{K}=5$ [5].

In this novel approach of detecting face, K-means have been used for segregating or separating the skin from the non-skin pixels. $\mathrm{K}$ used in this algorithm is 5 , as it gives the best result in our case.

Skin color segmented image contains only human skin like areas or regions, but we don't know that which region is having exact human face so, we need to send this image for further stage for rejection of non human face regions so that finally image contains only human face.

\subsection{Binary Image}

A binary image is of class logical. Binary images contain only 0 's and 1's. Pixels with the value 0 are displayed as black and pixels with the value 1 are displayed as white. Skin regions are shown as white (logical 1) in binary image. Once the skin area has been segmented we create a binary image from this. This binary image contains just 1 and 0 . Pixels with skin area are shown with 1 and pixels with non-skin area are shown with 0 . The skin color segmented image, is converted into binary image. Now, further operations are performed on this binary image.

\subsection{Morphological Operations}

Now, that we have the skin and non skin regions a series of morphological operations are performed to clean up the image. Various morphological operations are:

\subsubsection{Removal of small areas-}

In this we remove the small connected areas. This is used to remove small objects from the image while preserving the shape and size of larger objects in the image. In a binary image there may be areas which have small skin areas. So, by using this function all connected objects in a binary image are removed which have fewer than the required pixels, producing another binary image.

\subsubsection{Morphological opening -}

The morphological opening on binary image is performed with the structuring element. The definition of morphological opening of an image is erosion followed by dilation, using the same structuring element for both operations. A disk shaped structuring element is used in our case. 


\subsubsection{Hole Filling -}

To remove major holes in the image we use hole filling. It is done to keep the faces as single connected regions in anticipation of a second much larger morphological opening. Otherwise, the mask image will contain many cavities and holes in the faces.

\subsubsection{Morphological closing -}

The morphological closing on binary image is performed with the structuring element. The definition of morphological closing of an image is dilation followed by erosion, using the same structuring element for both operations. A disk shaped structuring element is used in our case.

\subsection{Rejection of non face skin regions}

According to the output of previous sections it is decided which region shows the skin so that a reliable search can be made in regions to find the face region. Here some numerical information about the regions is needed. For example, an assumption like "if there is a face in a region there must be at least one hole because of the eyes and nose" will prevent looking unrelated regions in image. It is assumed that if a region has at least one hole, then the region may contain a facial region and that region is processed to locate a face. Otherwise the region is ignored. The process of determining how many regions in a binary image is done by labeling such regions. A label is an integer value. An 8-connected neighborhood is used in order to determine the labeling of a pixel. If any of the neighbor pixels had a label, the current pixel is labeled with that label. If not, then a new label is used. At the end, the number of labels will be counted in the segmented image and this will be the number of regions. To separate each of these regions, the image is scanned and a new image is created that will have ones in the positions where the label occurs. The others are set to zero. After this, we iterate through each of the regions found in order to determine if the region might suggest a human face or not [1].

Now, for each of the connected regions or labeled regions we measure a set of properties like Euler no., eccentricity and bounding box.

\subsubsection{Holes in Region-}

For a skin to be face there must be some holes in it like nose, eyes etc. If there is no hole in the skin we can safely conclude that that area is not face. For calculating holes we find the Euler no of each region.

Number of holes in a region can be calculated by using Euler number of the region:

Where,

$$
\mathrm{E}=\mathrm{C}-\mathrm{H}
$$

E: The Euler number

$\mathrm{C}$ : The number of connected components

$\mathrm{H}$ : The number of holes in a region.

\subsubsection{Eccentricity -}

To classify if the detected region is face or not we check each of the region. The face is oval in shape. So, we have to reject all the regions which are not oval. For this we use the eccentricity based region property of the MATLAB. For each of the region we calculate the eccentricity. An ellipse (skin region) whose eccentricity is 0 is actually a circle, while an ellipse whose eccentricity is 1 is a line segment. The oval shape of a face can be approximated by an ellipse so we calculate Eccentricity of all skin connected regions. We discard all skin regions whose eccentricity is greater than 0.89905 as given by [8].

\subsubsection{Height to Width Ratio -}

In certain cases the largest bounding box consists of a perfect face. In order to isolate these regions we use dimension of the faces as a restriction. Now, of the selected regions left we calculate the height to width ratio of all regions. The width and the height of the region are used to improve the decision process. The height to width ratio of the human faces is around 1 . To include the variations of the face sizes and also to consider the fact that the neck region is very likely to be part of the connected components if exposed, we take this ratio between 0.8 and 1.6. If the ratio of any region lies between this it is taken as face [1].

\subsection{Detection of Face}

Based on the above criteria, we finally get the regions which can be classified as face. Now, we have to show the face in the original image. So, a bounding box of red color is drawn against the original image on the region identified as face only.

\section{PROPOSED FACE DETECTION ALGORITHM}

Step 1: Input image.

Step 2: Apply k-means for skin segmentation.

Step 3: Convert the image to binary image.

Step 4: To clean the image apply morphological operations on this image.

Step 5: Count the number of regions in the image by implementing connectivity analysis using 8-connected neighborhood.

Step 6: Find the number of holes in each region, using Euler no.

Step 7: If holes $>=1$ then region can be face, so move to next step else reject the region.

Step 8: For each region check eccentricity. If eccentricity is within the range then accept that region else reject the region.

Step 9: Calculate height and width for each region.

Step 10: For each region, if height to width ratio is within the range then the region is accepted as a face else it is rejected.

Step 11: Draw rectangle around the detected face.

\section{IMPLEMENTATION}

We have developed an algorithm that can detect human faces from an image. The algorithm has been successfully implemented on MATLAB R2008b. All the images have a size of $408 * 306$. Skin color has been taken as a tool for detection. The result on few images is shown in fig. 2 . The original images are shown in fig. 2(a). After the conversion of image into binary image with 0 and 1 values, we get images as shown in fig. 2(b). Finally, the detected face is shown in fig. 2(c) 

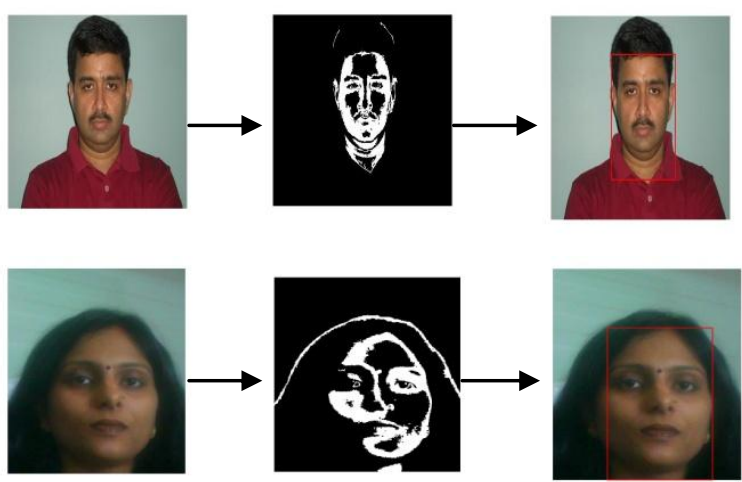

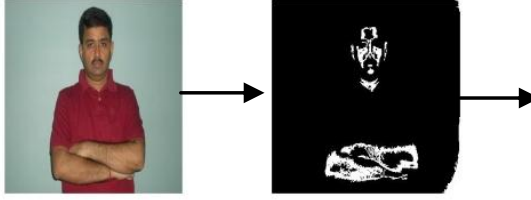

(a) (b)

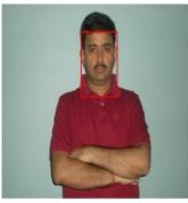

(c)
Fig 2: (a) The original image (b) The binary image (c) The detected face

\section{CONCLUSION}

In this paper, we have developed a novel frontal face detection technique. This is a robust algorithm that first separates skin regions from non skin regions, and efficiently locates faces within skin regions. This works well with the images we have taken and it very well segments the skin and non-skin area using color. This color segmentation forms the basis for final face detection. Finally the morphological operations and region property helps in detecting faces within the skin regions. The whole algorithm has been implemented on Matlab R2008b and it successfully draws a rectangle around the detected face region.

\section{REFERENCES}

[1] Nallaperumal K., Subban R., Krishnaveni.K, Fred L., Selvakumar K.R. Human Face Detection in Color Images Using Skin Color and Template Matching Models for Multimedia on the Web, IFIP International Conference on Wireless and Optical Communications Networks (IEEE), 5 pp, 7 August 2006.

[2] Anisetti M. Fast and robust face detection, Multimedia Techniques for Device and Ambient Intelligence (Springer US,), pp 43-72, 2009.

[3] Fasel R. I., Movellan R. J., A Comparison of Face Detection Algorithms, Proceedings of the International Conference on Artificial Neural Networks (Lecture Notes in Computer Science 2415 Springer 2002), pp 1325-1332, August 28-30, 2002.

[4] Farajzadeh N., Faez K. Hybrid Face Detection System with Robust Face and non-Face Discriminability , 23rd International Conference Image and Vision Computing New Zealand, pp 1-6, 2008.

[5] Fang X., Wenquan Gu W., Huang C. A Method of Skin Color Identification Based On Color Classification, International Conference on Computer Science and
Network Technology(IEEE), pp 2355 - 2358, 24-26 December 2011

[6] M.-H. Yang, Kriegman D., Ahuja N. Detecting Faces in Images: A Survey, IEEE Transactions on Pattern Analysis and Machine Intelligence, vo1.24, no.1 , pp 3458, Jan. 2002

[7] Hjelm E. and Low K.B. Face Detection: A survey, Computer Vision and Image Understanding, vol. 83,110.3, pp.236-274, Sept.2001.

[8] Raghuvanshi S.D., Agrawal D. Human Face Detection by using Skin Color Segmentation, Face Features and Regions Properties, International Journal of Computer Applications (0975 - 8887) Volume 38- No.9, January 2012.

[9] Thakur S., Paul S., Mondal A., Das S., Abraham A. Face Detection Using Skin Tone Segmentation, World Congress on Information and Communication Technologies (WICT), pp 53-60, 11-14 December, 2011.

[10] Mu-Chun Su, Chien-Hsing Chou, Modified Version of the K-Means Algorithm with a Distance Based on Cluster Symmetry, IEEE Transactions on Pattern Analysis and Machine Intelligence, Volume 23 Issue 6, pp 674-680, June 2001.

[11] Wen-Hui Lin, Jhen-Chih Liao, A Fast Face Segmentation Based On Color Spatial Features And KMeans Clustering Ensembles, Sixth International Conference on Machine Learning and Cybernetics (IEEE), Vol. 4, pp 1923 - 1929,19-22 August 2007.

[12] Zhang H., Zhao D., Gao W., Chen X. Combining Skin Color Model and Neural Network for Rotation Invariant Face Detection, Springer-Verlag Berlin Heidelberg, pp. 237-244, 2000.

[13] Singh K. S., Chauhan S.D., Vatsa M., Singh R. A Robust Skin Color Based Face Detection Algorithm, Tamkang Journal of Science and Engineering, Vol. 6, No. 4, pp 227-234, 2003.

[14] Ravi Kumar N.C., Bindu A., An Efficient Skin Illumination Compensation Model for Efficient Face Detection, IEEE Industrial Electronics Society Annual Conference, Vol. 1, pp 3444-3449, 6-10 November, 2006.

[15] Musa Z., Jumari K., Zainal N., A Hybrid Skin Detection for Surveillance System Based on Background Subtraction and Skin Color, Journal of Applied Sciences Research, vol 6, Issue 2, pp 1191-1199, February 2012. 